\title{
Satisfação do usuário do abastecimento de água em comunidades rurais em Boa Esperança, ES, Brasil
}

\begin{abstract}
A garantia do acesso à água segura nas áreas rurais é um objetivo que segue inatingido em diversas partes do mundo. No Brasil, o cenário é semelhante, apresentando um número elevado de instalações rurais sem abastecimento adequado de água. Em algumas comunidades rurais na cidade de Boa Esperança, ES, estabeleceu-se o modelo de gestão comunitária do abastecimento de água, também aplicado em outras partes do Brasil e de outros países. Neste trabalho, verificou-se a satisfação dos usuários destes sistemas em 7 destas comunidades. Com um questionário Likert, verificou-se o quanto os respondentes concordavam com cada uma de três afirmativas, contemplando os tópicos 'Qualidade da água', 'Quantidade de água' e 'Valor da tarifa'. Também foram levantados dados socioeconômicos daqueles que responderam e dados técnicos sobre as instalações do sistema, a comunidade e a associação de moradores. Observou-se que, no geral, os moradores estão satisfeitos com o abastecimento de água que recebem. Houve maior discordância sobre a qualidade da água, porém as respostas ainda foram majoritariamente positivas. Acredita-se que a população destas regiões não tenha informação suficiente sobre os padrões de qualidade da água. Desta maneira, é tão necessário conscientizar a população sobre estes padrões, quanto prover auxílio técnico para que elas os atinjam.
\end{abstract}

Palavras-chave: Saneamento Rural; Gestão Comunitária; Abastecimento de Água; Satisfação do Usuário; Brasil.

\section{User satisfaction with water supply services in rural communities in Boa Esperança, ES, Brazil}

\begin{abstract}
Ensuring access to safe water in rural areas is an objective that remains unattainable in various parts of the world. In Brazil, the scenario is similar, with a high number of rural facilities without adequate water supply. In some rural communities in the city of Boa Esperança, ES, the community water supply management model was established, also applied in other parts of Brazil and other countries. In this work, it was verified the satisfaction of the users of these systems in 7 of these communities. With a Likert questionnaire, it was verified how much the respondents agreed with each of three statements, covering the topics 'Water quality', 'Water quantity' and 'Value of the tariff'. Socio-economic data were also collected from those who responded and technical data on the system's facilities, the community and the residents' association. It was observed that, in general, the residents are satisfied with the water supply they receive. There was greate disagreement about water quality, but the responses were still mostly positive. It is believed that the population of these regions does not have sufficient information on water quality standards. Thus, it is as necessary to make the population aware of these standards, as well as to provide technical assistance so that they reach them.
\end{abstract}

Keywords: Rural Sanitation; Community Management; Water Supply; User Satisfaction; Brazil.

Anna Viginia Muniz Machado (iD Universidade Federal Fluminense, Brasil http://lattes.cnpq.br/9250702243182081 http://orcid.org/0000-0001-5793-8591 annav.machado@gmail.com

Pedro Antonio de Paiva Aranha (iD Universidade Federal Fluminense, Brasil http://lattes.cnpq.br/3501123938690633 http://orcid.org/0000-0002-6241-8934 pedroaranha@id.uff.br

Ricardo Pereira Gonçalves (D) Universidade Federal Fluminense, Brasil http://lattes.cnpq.br/8172965895049874 http://orcid.org/0000-0003-2185-8471 pereira.rlk@gmail.com



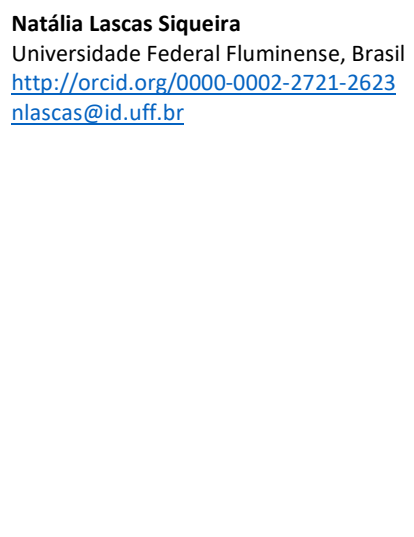

Referencing this:

MACHADO, A. V. M.; ARANHA, P. A. P.; GONÇALVES, R. P.; SILVA, J. C. D.; SANTOS, J. A. N.; SOUZA, A. C. S. V.; SIQUEIRA, N. L.. Satisfação do usuário do abastecimento de água em comunidades rurais em Boa Esperança, ES, Brasil. Revista Ibero Americana de Ciências Ambientais, v.12, n.1, p.322-334, 2021. DOI:

http://doi.org/10.6008/CBPC2179-6858.2021.001.0027 


\section{INTRODUÇÃO}

O acesso à água segura, estabelecido como um direito humano em 2010 (UNITED NATIONS, 2010), ainda não está efetivamente disseminado, especialmente em áreas rurais. Este cenário é influenciado tanto pelas condições geográficas dessas regiões, como sua distância aos centros urbanos (BARDE, 2017) por exemplo, quanto pela falta de investimento dos governos. De acordo com a Organização Mundial da Saúde (WHO, 2017), áreas urbanas recebem financiamentos três vezes maiores que as rurais para investimentos em água, saneamento e higiene. Este cenário se reflete no Brasil, onde 34,5\% das residências rurais têm abastecimento de água precário, ou ausente, de acordo com a Pesquisa Nacional por Amostra de Domicílios (IBGE, 2016b). Ressalta-se que estes dados levam em conta apenas o tipo de abastecimento nas residências e não a qualidade da água.

Há dificuldade de companhias privadas em receberem retorno de seus investimentos ao operarem em áreas onde a população não pode pagar por tarifas tradicionais de água (ALVES et al., 2016; ATAÍDE et al., 2012; CALZADA et al., 2017). Sendo essa a realidade de muitas comunidades rurais, o modelo de gestão comunitária tem sido uma alternativa comumente adotada em diversos países, em áreas com tais características (CALZADA et al., 2017; HELLER et al., 2006; WHITTINGTON et al., 2009). Neste modelo, a população se torna responsável por operar e gerir o sistema de abastecimento de água, por meio de uma associação de moradores. A construção das estações, bem como a prestação de assistência técnica, institucional e política é delegada a agentes externos, como prefeituras e governos dos estados (BEHNKE et al., 2017; HUNTER et al., 2010; HUTCHINGS et al., 2015; KLEEMEIER, 2000; WHITTINGTON et al., 2009).

Esse modelo de gestão vai ao encontro dos Objetivos de Desenvolvimento Sustentável (ODS), em particular ao objetivo 6, "Assegurar a disponibilidade e gestão sustentável da água e saneamento para todas e todos". Mais especificamente aos itens 6.5, "Até 2030, implementar a gestão integrada dos recursos hídricos em todos os níveis, inclusive via cooperação transfronteiriça, conforme apropriado" e 6.b "Apoiar e fortalecer a participação das comunidades locais, para melhorar a gestão da água e do saneamento" (UNITED NATIONS, 2015).

O sistema de gestão comunitária de água tem mostrado, tanto casos de sucesso, com sistemas considerados sustentáveis, quanto casos onde não se verifica a sustentabilidade (MACHADO et al., 2020). Em geral, a verificação da possibilidade de sustentabilidade de sistemas de abastecimento de água tem focado em critérios técnicos, de gestão e na efetividade de políticas regulatórias (BHANDARI et al., 2007; KUMASI et al., 2018; SPALING et al., 2014). Ainda de acordo com os autores, embora esses critérios sejam relevantes para entender o potencial de sustentabilidade dos sistemas, eles não são suficientes. Deve-se levar em conta também a satisfação dos usuários, pois está implica na disposição da população em pagar pelos serviços continuamente, um fator fundamental para a sustentabilidade do sistema (ALDERWISH et al., 2013; BHANDARI et al., 2007; KELLY et al., 2017; MACHADO et al., 2019; RAUTANEN et al., 2018; SARA et al., 2005; TIGABU et al., 2013).

Este estudo se propôs a verificar o nível de satisfação dos usuários de sistemas de abastecimento de 
água sob gestão comunitária e comparar os resultados com dados de levantamentos técnicos realizados na área de estudo. Para este trabalho foram escolhidas sete comunidades no município de Boa Esperança, no estado do Espírito Santo, Brasil. Foi aplicado um questionário utilizando a escala Likert (LIKERT, 1932), a fim de capturar a percepção dos usuários sobre a qualidade da água, a quantidade de água e tarifa do serviço de abastecimento de água. Para o levantamento técnico, dados foram coletados e avaliados segundo a metodologia SIASAR (2012).

\section{METODOLOGIA}

\section{Área de Estudo}

O estudo foi conduzido em sete comunidades rurais no município de Boa Esperança, a noroeste do estado do Espírito Santo, na região sudeste do Brasil. O município tem extensão de aproximadamente $428,716 \mathrm{~km}^{2}$ (IBGE, 2011) e compreende vinte e três comunidades rurais, dentre as quais, sete foram objetos de coleta de dados. Boa Esperança está localizado a uma latitude $18^{\circ} 32^{\prime} 24^{\prime \prime}$ S e a uma longitude $40^{\circ} 17^{\prime} 45^{\prime \prime W}$, estando a 140 metros acima do nível do mar. A Figura 1 ilustra o município de Boa Esperança, destacando as comunidades estudadas.

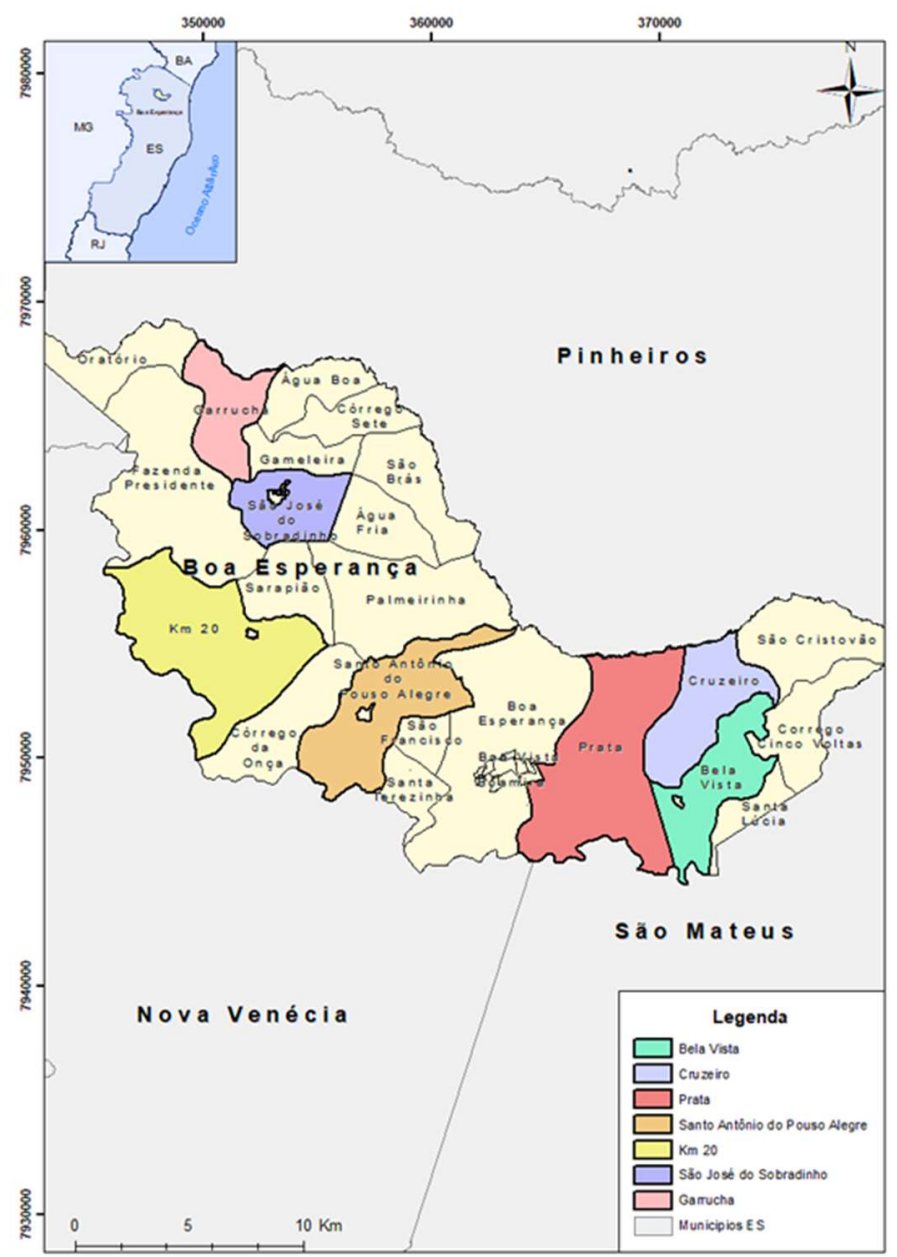

Figura 1: Município de Boa Esperança, Espírito Santo, Brasil.

O município tem relevo majoritariamente plano e clima quente e seco. Apesar do clima tropical, Boa 
Esperança tem regiões de clima semiárido ao seu entorno e apenas três meses do ano, janeiro, novembro e dezembro, são considerados chuvosos. Desta forma, a região é considerada propensa à seca e à desertificação (ESPÍRITO SANTO, 2011).

A população do município, foi estimada em 15390 habitantes no ano do estudo (IBGE, 2016a) e está distribuída 72,11\% em áreas urbanas, e 27,89\% em áreas rurais, 50,56\% são homens e 49,44\% mulheres (IBGE, 2011). O Índice de Desenvolvimento Humano Municipal foi avaliado em 0,679, ocupando, desta forma, a 45a posição entre os 78 municípios do estado (PNUD et al., 2013). Sua estrutura fundiária é predominantemente composta por pequenas propriedades, onde é realizado trabalho agrícola familiar ou cooperativo. A cultura do café ocupa a maior área das terras onde há produção agrícola (ESPÍRITO SANTO, 2011).

Para o levantamento de comunidades rurais foi utilizada a base de dados Sistema Integrado de Bases Geoespaciais do Estado do Espírito Santo - GEOBASES (ESPÍRITO SANTO, 2016), através da interface 'Navegador Público'. Usando a camada 'Comunidades Urbanas e Rurais', foram consideradas as comunidades pertencentes ao município de Boa Esperança, classificadas em situação rural. Estas comunidades foram então exportadas para uma planilha, na qual foram selecionadas aleatoriamente 10 dentre o total de 23 . Foram excluídas 3 comunidades por motivos logísticos, como a não possibilidade de realizar contato com representantes das associações de moradores ou dificuldades de acesso às estações de coleta e/ou tratamento.

A Tabela 1 indica as comunidades estudadas, a área destas, o número de ligações ativas no sistema de abastecimento, e o número de formulários de satisfação do usuário aplicados em cada uma.

Tabela 1: Relação das comunidades estudadas, suas áreas, número de ligações ativas e de formulários de satisfação do usuário aplicados no estudo.

\begin{tabular}{llll}
\hline Comunidade & Área $\left(\mathrm{km}^{2}\right)$ & Ligações Ativas & Formulários Aplicados \\
\hline Km 20 & 39,6394 & 146 & 12 \\
Garrucha & 13,4957 & 32 & 3 \\
Bela Vista & 18,3655 & 64 & 4 \\
Prata & 41,3329 & 10 & 1 \\
Cruzeiro & 18,2652 & 10 & 2 \\
Santo Antônio do Pouso & 26,8811 & 370 & 15 \\
Alegre & 12,7519 & 285 & 14 \\
São José do Sobradinho & 170,7317 & 917 & 51 \\
\hline Total & & & \\
\hline
\end{tabular}

Fontes: GEOBASES (área).

\section{Coleta de Dados}

\section{Percepção do Usuário e Dados Socioeconômicos}

Para o levantamento de dados sobre a percepção dos usuários, foi conduzida uma entrevista estruturada, com uma relação fixa de perguntas para todos os entrevistados nos dias 18 e 19 de agosto de 2016, em cada uma das comunidades estudadas. Um formulário utilizando a escala Likert foi usado, este modelo de questionário foi escolhido por ser bem estabelecido em pesquisas sociais e de saúde pública e comum em pesquisas de opinião, além de permitir uma avaliação quantitativa dos resultados. Um segundo questionário foi aplicado com objetivo de levantar o perfil socioeconômico dos entrevistados, bem como sua 
participação ou não em comitê ou associação da água de sua comunidade.

Para a seleção dos entrevistados, foi aplicada amostragem por conveniência. Consideraram-se moradores que estavam nas ruas, ou em residências próximas aos locais de visita dos entrevistadores. Foram realizadas entrevistas apenas com aqueles que estavam dispostos a responder e eram usuários do sistema de abastecimento estudado.

As entrevistas foram conduzidas oralmente, visando a inclusão de respondentes não alfabetizados. Foi informado aos entrevistados, através de um termo de consentimento, que seus nomes não seriam revelados em conjunto com suas falas e que a entrevista não envolveria nenhum tipo de pagamento. 0 termo foi entregue aos respondentes para que fosse lido e assinado e, alternativamente, o termo foi lido pelo entrevistador aos respondentes não alfabetizados e assinado por um membro da família. As entrevistadoras e a pesquisadora responsável também assinaram os termos.

O formulário de percepção do usuário está reproduzido no Quadro 1. Ele consiste em três afirmativas, cada uma sobre um dos seguintes temas: qualidade de água, quantidade de água e tarifa do serviço de abastecimento de água. Os entrevistados foram convidados a dar suas opiniões de acordo com as 6 possíveis respostas, a saber: 'Discordo completamente'; 'Discordo'; 'Não concordo, nem discordo'; 'Concordo'; 'Concordo completamente'; 'Não entendi a afirmação', representadas no Quadro 1 pelas siglas 'DC'; 'D'; 'N'; 'C'; 'CC'; 'NE', respetivamente.

Quadro 1: Afirmativas para definição da satisfação do usuário.

\begin{tabular}{|c|c|c|c|c|c|c|c|}
\hline \multicolumn{2}{|c|}{1 - Satisfação do Usuário } & DC & $\mathrm{D}$ & $\mathrm{N}$ & $\mathrm{C}$ & $\mathrm{CC}$ & $\mathrm{NE}$ \\
\hline 1.1- & A qualidade da água é satisfatória para uso doméstico. & & & & & & \\
\hline $1.2-$ & A quantidade da água é suficiente para o abastecimento doméstico. & & & & & & \\
\hline $1.3-$ & O valor da conta é compatível com o serviço. & & & & & & \\
\hline
\end{tabular}

O formulário utilizado para capturar os dados do usuário está reproduzido no Quadro 2. As perguntas $1,3,4,5,9,11,12,13,14$ e 15 foram respondidas levando-se em consideração as opções de respostas previamente definidas, conforme reproduzidas no quadro. As demais aceitaram respostas livres, sendo todas numéricas, exceto à pergunta número 6 que foi respondida com o nome da comunidade onde residia o respondente.

Quadro 2: Formulário para o levantamento de dados dos usuários.

\begin{tabular}{|c|c|}
\hline Dado & Possíveis respostas (quando há) \\
\hline 1 - Grau de escolaridade & 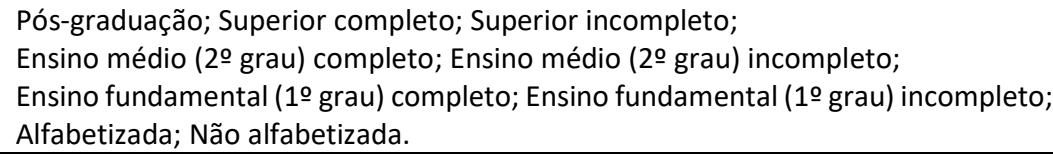 \\
\hline \multicolumn{2}{|r|}{ (2: } \\
\hline 3 - Gênero & Masculino; Feminino; Prefiro não informar. \\
\hline $\begin{array}{l}4 \text { - Participa do comitê ou associação da } \\
\text { água? }\end{array}$ & Sim; Não. \\
\hline $\begin{array}{l}5 \text { - Distância da residência à estação de } \\
\text { tratamento }\end{array}$ & Até 1 km; Até 2 km; Até 3 km; Até 4 km; Até 5 km; Mais que 5 km. \\
\hline \multicolumn{2}{|l|}{6 - Em que comunidade você reside? } \\
\hline \multicolumn{2}{|l|}{7 - Quanto tempo mora na comunidade? } \\
\hline \multicolumn{2}{|l|}{$\begin{array}{l}8 \text { - Quantos moradores residem no } \\
\text { domicílio? }\end{array}$} \\
\hline $\begin{array}{l}9 \text { - Qual a renda familiar? } \\
\text { (medido em salários mínimos) }\end{array}$ & Até 1; Até 2; Até 3; Até 4; Até 5; Mais de 5. \\
\hline
\end{tabular}




\begin{tabular}{|l|l|}
\hline 10 - Qual o valor aproximado da sua conta? & \\
\hline 11 - O domicílio possui telefone fixo? & Sim; Não. \\
\hline $\begin{array}{l}12 \text { - Algum morador do domicílio possui } \\
\text { telefone celular? }\end{array}$ & Sim; Não. \\
\hline 13 - Possui acesso à internet? & Sim; Não. \\
\hline 14 - Possui TV? & Sim; Não. \\
\hline 15 - Possui TV a cabo? & Sim; Não. \\
\hline
\end{tabular}

\section{Dados Técnicos}

Os dados técnicos foram levantados em entrevistas estruturadas, segundo a metodologia Sistema de Informação de Água e Saneamento Rural (SIASAR) nos dias 18 e 19 de agosto de 2016. A metodologia SIASAR foi escolhida por ter sido construída com o propósito exclusivo de analisar o abastecimento de água e saneamento em comunidades rurais, e ser usada por países como Honduras, Nicarágua, Panamá, República Dominicana, Costa Rica, Peru, Bolívia, Paraguai e Quirguistão, além dos estados de Oaxaca (México) e Ceará (Brasil) para o monitoramento das condições de água e saneamento locais (SIASAR, 2017).

A metodologia consiste em entrevistas com representantes das associações ou comitês de água das comunidades para preencher quatro questionários: Questionário de Comunidade; Questionário de Sistema; Questionário de Prestador de Serviço; Questionário de Prestador de Assistência Técnica. O Protocolo de Campo (SIASAR, 2017) define: 1. Comunidade: Assentamento humano de caráter rural; 2. Sistema: A infraestrutura de maior ordem na comunidade cujo fim é prover água potável; 3. Prestador de Serviço: Um organismo local, ou estatal, responsável por oferecer o serviço de água para a comunidade; 4. Prestador de Assistência Técnica: Entidade ou organização que provê assistência técnica ao prestador de serviço.

As entrevistas foram conduzidas com presidentes das associações de moradores, se existentes. $\mathrm{Na}$ sua ausência ou na necessidade de obtenção de informações mais apuradas também foram considerados os operadores do sistema. Exclusivamente na comunidade de Garrucha, o ex-presidente da associação de moradores foi convidado para entrevista. As entrevistas foram conduzidas oralmente. Uma autorização para visita técnica, coleta e utilização de dados foi assinada pelos entrevistados e pelos pesquisadores. Medições e observações também foram realizadas pelos pesquisadores nas instalações dos sistemas, a fim de levantar as informações necessárias para o preenchimento dos formulários. A aplicação do SIASAR nestas e em outras comunidades rurais, e seus resultados, estão descritos em maiores detalhes em Machado et al. (2020).

\section{Processamento de Dados}

\section{Percepção do Usuário e Confiabilidade do Questionário}

As respostas, segundo o modelo Likert, foram convertidas em pontuações inteiras de 1 a 6 , sendo estas correspondentes a 'Discordo completamente' e 'Concordo completamente', respectivamente. Desta maneira pode-se verificar a média e o desvio padrão destes itens.

A confiabilidade do questionário que avaliou a percepção do usuário foi avaliada com o cálculo do Coeficiente Alfa de Cronbach realizado com o software SPSS Data Editor. Segundo Hair et al. (2009), este coeficiente é uma medida de confiabilidade que varia de 0 a 1, conforme aumenta a confiabilidade. Os valores obtidos nesta pesquisa são considerados aceitáveis, pois encontram-se no limite inferior de 
aceitabilidade $(0,60$ e 0,70), conforme observa-se no Quadro 3.

Quadro 3: Estatísticas de confiabilidade.

\begin{tabular}{|l|l|l|}
\hline Alfa de Cronbach & Alfa de Cronbach com base em itens padronizados & № de itens \\
\hline 0,686 & 0,728 & 3 \\
\hline
\end{tabular}

Ainda se utilizando o software SPSS, foi avaliada a validade de construto por meio da análise fatorial exploratória das variáveis estudadas, incluindo o Teste de KMO (Medida Kaiser-Meyer-Olkin de adequação de amostragem) e o Teste de esfericidade de Bartlett. Os resultados são considerados suficientes por Hair et al. (2009) e Field (2009), e estão reproduzidos no Quadro 4.

Quadro 4: Teste de KMO e Bartlett.

\begin{tabular}{|l|l|l|}
\hline Medida Kaiser-Meyer-Olkin de adequação de amostragem. & 0,584 \\
\hline Teste de esfericidade de Bartlett & Qui-quadrado aprox. & 38,100 \\
\cline { 2 - 3 } & df & 3 \\
\cline { 2 - 3 } & Sig. & 0,000 \\
\hline
\end{tabular}

\section{Dados Socioeconômicos}

Os dados coletados através do questionário socioeconômico foram tabulados em planilhas, e em seguida foram processados de maneira descritiva. Foram calculadas médias para as variáveis idade, tempo de moradia na comunidade, renda familiar e valor da conta. Para as variáveis sexo feminino, participação na associação da água, grau de escolaridade, domicílios com telefone celular, domicílios com internet e domicílios com TV foram calculadas porcentagens em relação ao total $(n=51)$.

\section{Dados técnicos}

O levantamento realizado com o SIASAR foi tabulado em uma planilha e avaliado seguindo os critérios da própria metodologia. A partir dos dados coletados sobre cada um dos atores (Comunidade, Sistema, Prestador de Serviço e Prestador de Assistência Técnica) são atribuídas notas de acordo com critérios devidamente definidos pela metodologia. Devido à grande quantidade de fatores analisados para cada ator, estes não serão reproduzidos aqui, porém podem ser consultados na referência (SIASAR, 2012). Após a atribuição das classificações, as notas de cada ator podem ser interpretadas de acordo com o Quadro 5.

Quadro 5: Interpretação da Classificação de Atores obtida com o SIASAR.

\begin{tabular}{|c|c|c|c|c|}
\hline \multirow{2}{*}{ Entidades } & \multicolumn{4}{|l|}{ Classificação } \\
\hline & $\mathrm{A}$ & $\mathrm{B}$ & $\mathrm{C}$ & $\mathrm{D}$ \\
\hline Comunidade & $\begin{array}{l}\text { A comunidade possui } \\
\text { ambiente sadio e a } \\
\text { cobertura de água e } \\
\text { saneamento é } \\
\text { pertinente }\end{array}$ & $\begin{array}{l}\text { O serviço de água e } \\
\text { saneamento da } \\
\text { comunidade não é } \\
\text { completo }\end{array}$ & $\begin{array}{l}\text { A comunidade possui } \\
\text { sérios problemas na } \\
\text { cobertura de água e } \\
\text { esgoto }\end{array}$ & $\begin{array}{l}\text { A comunidade possui } \\
\text { problemas ambientais } \\
\text { graves e a cobertura é } \\
\text { baixíssima }\end{array}$ \\
\hline Sistema & $\begin{array}{l}\text { O sistema funciona } \\
\text { perfeitamente }\end{array}$ & $\begin{array}{l}\text { O sistema possui mal } \\
\text { funcionamento ou } \\
\text { falhas reparáveis pelo } \\
\text { prestador }\end{array}$ & $\begin{array}{l}\text { O sistema possui graves } \\
\text { falhas, nas quais o } \\
\text { prestador não é apto } \\
\text { para consertar }\end{array}$ & O sistema é inoperante \\
\hline
\end{tabular}




\begin{tabular}{|c|c|c|c|c|}
\hline Prestador de serviço & $\begin{array}{l}\text { O prestador do } \\
\text { serviço garante } \\
\text { sustentabilidade } \\
\text { possui } \\
\text { organização }\end{array}$ & $\begin{array}{l}\text { O prestador mantém } \\
\text { sustentabilidade, } \\
\text { porém possui } \\
\text { organização mediana }\end{array}$ & $\begin{array}{l}\text { O prestador do serviço } \\
\text { não é sustentável e não } \\
\text { possui boa organização }\end{array}$ & $\begin{array}{l}\text { O prestador do serviço está } \\
\text { inoperante e o sistema está } \\
\text { a ponto de ruir }\end{array}$ \\
\hline $\begin{array}{l}\text { Prestador de } \\
\text { assistência técnica }\end{array}$ & $\begin{array}{l}\text { O prestador do } \\
\text { serviço está } \\
\text { desempenhando o } \\
\text { serviço } \\
\text { adequadamente seu } \\
\text { trabalho e/ou possui } \\
\text { recursos suficientes }\end{array}$ & $\begin{array}{l}\text { O prestador de } \\
\text { assistência técnica } \\
\text { não apoia totalmente } \\
\text { e/ou possui recursos } \\
\text { limitados }\end{array}$ & $\begin{array}{l}0 \text { prestador de } \\
\text { assistência apenas } \\
\text { cumpre sua função e/ou } \\
\text { carece de recursos }\end{array}$ & $\begin{array}{l}\text { O prestador de assistência } \\
\text { técnica não está prestando } \\
\text { serviço e/ou não possui } \\
\text { recursos atribuídos }\end{array}$ \\
\hline
\end{tabular}

Fonte: SIASAR (2012).

O SIASAR também gera um Índice de Sustentabilidade dos Serviços de Abastecimento de Água (ISSA). Este índice é calculado a partir dos seguintes parâmetros: número de casas abastecidas pelo sistema $\left(n_{c}\right)$, número de casas na comunidade $\left(n_{t}\right)$ e pesos associados às classificações do sistema $\left(p_{s}\right)$ e do prestador de serviço $\left(p_{p}\right)$. A Equação 1 descreve a fórmula para o cálculo do ISSA, que varia de 0 a 1 (quanto mais próximo de 1, maior a sustentabilidade do sistema). O Quadro 6 apresenta os pesos atribuídos ao prestador de serviço e o sistema de acordo com suas classificações.

$$
I S S A=\frac{n_{c} \times p_{s} \times p_{p}}{n_{t}}
$$

Quadro 6: Pesos para o cálculo do ISSA de acordo com a classificação SIASAR.

\begin{tabular}{|l|l|}
\hline Classificação & Peso \\
\hline A & 1,00 \\
\hline B & 0,66 \\
\hline D & 0,33 \\
\hline
\end{tabular}

Fonte: SIASAR (2012).

\section{RESULTADOS}

\section{Perfil socioeconômico dos entrevistados}

Nas setes comunidades visitadas, houve um total de 51 respondentes, com faixa etária entre 40 e 50 anos, sendo 27 indivíduos do sexo feminino, representando mais de 50\% dos entrevistados. Apenas um respondente participava de alguma associação ou comitê da água. Ademais, é importante ressaltar que todos os entrevistados possuem eletricidade em suas residências, assim como acesso a escolas e a unidades básicas de saúde. Outras informações coletadas sobre o perfil sociodemográfico dos respondentes podem ser observadas na Tabela 2.

Tabela 2: Perfil socioeconômico dos entrevistados.

\begin{tabular}{ll}
\hline Dado levantado & Resultado \\
\hline Número de respondentes & 51 \\
Idade média & 44,16 anos \\
Sexo feminino n (\%) & $27(52,94 \%)$ \\
Grau de escolaridade: & - \\
- Pós-graduação & $0,00 \%$ \\
- Superior completo & $0,00 \%$ \\
- Superior incompleto & $0,00 \%$ \\
- Ensino médio completo & $17,65 \%$ \\
- Ensino médio incompleto & $7,84 \%$ \\
- Ensino fundamental completo & $9,80 \%$ \\
- Ensino fundamental incompleto & $50,98 \%$
\end{tabular}


Alfabetizada

_ Não alfabetizada

Tempo médio de estadia na comunidade

Renda média familiar

Valor médio da conta

Domicílios com telefone celular

Domicílios com internet

Domicílios com TV
$5,88 \%$

$7,84 \%$

19,43 anos

1,53 salários mínimos

$\mathrm{R} \$ 21,02$

$100,00 \%$

$35,29 \%$

$94,12 \%$

\section{Percepção do usuário}

Os resultados do questionário sobre a percepção dos usuários quanto aos fatores que foram questionados estão reproduzidos na Tabela 3. Mais de 70\% dos entrevistados responderam que concordam completamente com a afirmativa 'A qualidade da água é satisfatória para o uso doméstico'. Se somados aos que responderam 'Concordo', a porcentagem de entrevistados que demonstrou uma opinião positiva sobre a qualidade da água ultrapassa os $88 \%$. Analisando-se as respostas sobre 'A quantidade de água é suficiente para o abastecimento doméstico', observa-se que cerca de $90 \%$ dos entrevistados concordam completamente, somando-se aos que concordam, $98 \%$ dos entrevistados demonstraram opinião positiva sobre este fator. Esta mesma porcentagem é encontrada quando se considera aqueles que concordam completamente (mais de $88 \%$ ) ou apenas concordam (próximo a 10\%) com a afirmativa 'O valor da conta é compatível com o serviço'.

Tabela 3: Resultados das afirmativas.

\begin{tabular}{|c|c|c|c|c|}
\hline & $\begin{array}{l}\text { A qualidade } \\
\text { satisfatória } \\
\text { doméstico. }\end{array}$ & $\begin{array}{l}\text { da água é } \\
\text { para uso }\end{array}$ & $\begin{array}{l}\text { A quantidade de água é suficiente para } \\
\text { o abastecimento doméstico. }\end{array}$ & $\begin{array}{l}\text { O valor da conta é } \\
\text { compatível com o serviço. }\end{array}$ \\
\hline $\begin{array}{l}\text { Discordo } \\
\text { completamente. }\end{array}$ & $0,00 \%$ & & $0,00 \%$ & $0,00 \%$ \\
\hline Discordo. & $1,96 \%$ & & $1,96 \%$ & $1,96 \%$ \\
\hline $\begin{array}{l}\text { Nem concordo nem } \\
\text { discordo. }\end{array}$ & $9,80 \%$ & & $0,00 \%$ & $0,00 \%$ \\
\hline Concordo. & $17,65 \%$ & & $7,84 \%$ & $9,80 \%$ \\
\hline $\begin{array}{l}\text { Concordo } \\
\text { completamente. }\end{array}$ & $70,59 \%$ & & $90,20 \%$ & $88,24 \%$ \\
\hline $\begin{array}{l}\text { Não entendi a } \\
\text { afirmação. }\end{array}$ & $0,00 \%$ & & $0,00 \%$ & $0,00 \%$ \\
\hline Total & $100,00 \%$ & & $100,00 \%$ & $100,00 \%$ \\
\hline
\end{tabular}

Cada item pode variar entre 1 (Discordo completamente) e 6 (Concordo completamente). 0 fator que apresentou maior desvio padrão foi a percepção sobre a qualidade da água, o que deve indicar que entre os entrevistados, há maior discordância sobre este item do que os outros. Em geral, houve pouca dispersão dos resultados. A Tabela 4 indica os valores numéricos de média e desvio padrão para cada variável. Já a Figura 2, mostra como as médias se aproximam das respostas.

Tabela 4: Valores de média e desvio padrão obtidos ( $N=51)$

\begin{tabular}{llll} 
& $\begin{array}{l}\text { A qualidade da água é satisfatória } \\
\text { para uso doméstico. }\end{array}$ & $\begin{array}{l}\text { A quantidade de água é suficiente para o } \\
\text { abastecimento doméstico }\end{array}$ & $\begin{array}{l}\text { O valor da conta é compatível } \\
\text { com o serviço. }\end{array}$ \\
\hline Média & 4,570 & 4,860 & 4,840 \\
Desvio & 0,755 & 0,491 & 0,505 \\
padrão & & & \\
\hline
\end{tabular}






Figura 2: Resultado das questões formuladas considerando intervalo de confiança de $95 \%$.

\section{Avaliação do SIASAR}

As sete comunidades estudadas foram avaliadas com o SIASAR. Em nenhuma das comunidades havia um prestador de assistência técnica fixo, com atuação regular. Desta maneira, não foi possível conduzir as entrevistas para a avaliação do ator Prestador de Assistência Técnica. Como não havia sistema de tratamento em Cruzeiro, este item não foi avaliado e o peso do Sistema foi tomado como 0. Prata e Cruzeiro tiveram ISSA avaliado em 0, devido aos pesos que receberam (vide Fórmula 1 e Quadro 6). As classificações dos atores Sistema, Prestador de Serviço e Comunidade, bem como o Índice de Sustentabilidade dos Serviços de Abastecimento de Água estão reproduzidos na Tabela 5. Particularidades sobre as comunidades serão levantadas ao longo da discussão com fim de permitir uma análise detalhada.

Tabela 5: Classificação e ISSA de acordo com o SIASAR.

\begin{tabular}{lllll}
\hline & Sistema & Prestador de Serviço & Comunidade & ISSA \\
\hline $\begin{array}{l}\text { Santo Antônio do Pouso } \\
\text { Alegre }\end{array}$ & B & B & A & 0,44 \\
Bela Vista & A & B & B & 0,66 \\
Garrucha & A & B & B & 0,66 \\
KM 20 & B & C & B & 0,22 \\
São José do Sobradinho & B & B & B & 0,44 \\
Prata & B & D & C & 0,00 \\
Cruzeiro & - & D & D & 0,00 \\
\hline
\end{tabular}

\section{DISCUSSÃO}

A qualidade de água foi o tópico com maior discordância, e com maior número de respostas não positivas. Cinco usuários responderam com 'Não concordo nem discordo' à afirmativa 'A qualidade da água é satisfatória'. Destes, quatro são moradores da comunidade de Santo Antônio do Pouso Alegre, e apenas um da comunidade de São José do Sobradinho. Com um ISSA de 0,44, estas comunidades não apresentam nem os melhores, nem os piores índices de sustentabilidade. Os dados técnicos indicam que os sistemas 
possuem mal funcionamento ou falhas reparáveis pelos prestadores de assistência técnica e os prestadores de serviço mantém sustentabilidade, porém possuem organização mediana. No caso de São José, observouse que o serviço de água e saneamento da comunidade não é completo, o que pode afetar diretamente a qualidade da água. Porém não se identificou isso em Santo Antônio. Essas comunidades não possuem áreas verdes ou protegidas no entorno da captação e estão sujeitas à poluição por resíduos agrícolas. As comunidades com maior ISSA, Bela Vista e Garrucha, apresentam áreas verdes e protegidas no entorno da captação, mas ainda estão sujeitas à contaminação por resíduos agrícolas. Nessas comunidades, todos responderam com 'Concordo completamente' à afirmativa que diz respeito à qualidade da água.

Sobradinho, Prata e Km 20 praticam tarifas fixas, enquanto Santo Antônio, Bela Vista e Garrucha praticavam valores fixos para o consumo até 10000 litros, cobrando um valor adicional para cada 1000 litros excedentes. Apenas quatro entrevistados informaram pagar acima do valor mínimo, porém todos estes concordam completamente com a afirmativa 'O valor da conta é compatível com o serviço'. $\mathrm{O}$ fato de a maioria dos entrevistados ter reportado pagar a taxa mínima, aliado ao alto índice de satisfação, deve indicar que esta foi bem definida de acordo com o consumo e as particularidades locais. A tarifa básica mais alta foi encontrada em Km 20 e Sobradinho, onde é de R\$ 25,00. Mas em Santo Antônio, onde a tarifa básica é R\$ 20,00 e a adicional $R \$ 2,00$ por cada 1000 litros, três usuários disseram pagar entre $R \$ 20,00$ e $R \$ 30,00$ todo mês. A tarifa mais baixa foi a de Prata, apenas $R \$$ 5,00. Embora existente, a inadimplência não afetou o funcionamento dos sistemas das comunidades, dada sua baixa incidência. Em $\mathrm{Km} \mathrm{20}$, foi reportado que alguns moradores pagam a cada dois meses, em acordo com a associação. Observa-se mais um caso de usuários satisfeitos e dispostos a pagar, reforçando a ideia de que a satisfação do usuário influencia na disposição a pagar.

Em geral, os usuários se mostraram satisfeitos com o serviço de abastecimento de água, mesmo que estes tenham tido uma performance não tão boa na avaliação técnica. Esse resultado, está alinhado com estudos anteriores, como: Magara (2014) que mostrou, no geral, usuários satisfeitos com os serviços, mesmo quando $70 \%$ dos entrevistados recebiam serviço abaixo das normas quanto à qualidade e quantidade em Uganda; Kumasi et al. (2015) observou usuários em determinados distritos de Gana que não se mostraram preocupados quanto a qualidade de água que recebiam; Kumasi et al. (2018) obteve resultados semelhantes, onde mostrou alto nível de satisfação entre usuários de sistemas que não atendiam ao padrão nacional de Gana. Os autores citados, questionam se os padrões de qualidade para o serviço rural de abastecimento de água são demasiado altos ou se os usuários apenas estão satisfeitos pois não têm conhecimento destes. É importante ressaltar que padrões elevados sobre o abastecimento de água têm influência direta na saúde da população local, e afetam indiretamente outras áreas do desenvolvimento local. Há um desafio no sentido de balancear o padrão de qualidade sob o qual as metodologias avaliam estes sistemas de pequeno porte, e o que se observa sobre a satisfação dos usuários.

É necessário que, ao mesmo tempo que os prestadores de serviço se esforcem para atingir os padrões de qualidade e quantidade, também trabalhem para a informação da comunidade sobre estes padrões. Porém, estas não são simples tarefas para os prestadores de serviço, visto que se tratam de organizações 
comunitárias compostas principalmente por voluntários, muitas vezes com poucos membros. Neste sentido, é importante a contribuição de agentes externos, como prefeituras, a fim de prover assistência técnica, institucional e política às organizações.

\section{CONCLUSÕES}

Foi verificado que nas sete comunidades estudadas, Km 20, Garrucha, Bela Vista, Prata, Cruzeiro, Santo Antônio do Pouso Alegre e São José do Sobradinho, os usuários dos sistemas de abastecimento de água sob gestão comunitária se encontram, no geral, satisfeitos. Os entrevistados se mostraram altamente favoráveis à quantidade de água recebida e ao valor da tarifa cobrada. Mostraram também uma percepção positiva, porém um pouco mais variada, sobre a qualidade da água recebida. Em contraste com os dados levantados segundo a metodologia SIASAR, a satisfação dos usuários se mostrou mais alta que a avaliação técnica do sistema.

\section{REFERÊNCIAS}

ALDERWISH, A. M.; DOTTRIDGE, J.. Evaluation of user satisfaction of rural water supply in Yemen. Journal of Water Sanitation and Hygiene for Development, v.3, n.3, p.322329, 2013. DOI: http://doi.org/10.2166/washdev.2013.067

ALVES, F. G. C.; ARAÚJO, F. T. V.. Sistemas de abastecimento em comunidades rurais do semiárido: a implantação do SISAR em Cristais, Cascavel, CE. Revista Tecnologia, v.37, n.1-2, p.78-86, 2016. DOI:

http://doi.org/10.5020/23180730.2016.v37.1/2.78-86

ATAÍDE, G. V. T. L.; MORAES, L. R. S.; BORJA, P. C..

Autogestão em saneamento básico no Brasil: experiências e aprendizado. In: EXPOSIÇÃO DE EXPERIÊNCIAS MUNICIPAIS EM SANEAMENTO, 16; SANEAMENTO BÁSICO: DESAFIOS E CENÁRIO PARA A UNIVERSALIZAÇÃO. Anais. Maringá, 2012. p.1-13.

BARDE, J. A.. What Determines Access to Piped Water in Rural Areas? Evidence from Small-Scale Supply Systems in Rural Brazil. World Development, v.95, p.88-110, 2017. DOI: http://dx.doi.org/10.1016/j.worlddev.2017.02.012

BEHNKE, N. L.; KLUG, T.; CRONK, R.; SHIELDS, K. F.; LEE, K.; KELLY, E. R.; ALLGOOD, G.; BARTRAM, J.. Resource mobilization for community-managed rural water systems: Evidence from Ghana, Kenya, and Zambia. Journal of Cleaner Production, v.156, p.437-444, 2017. DOI: http://dx.doi.org/10.1016/i.jclepro.2017.04.016

BHANDARI, B.; GRANT, M.. User satisfaction and sustainability of drinking water schemes in rural communities of Nepal. Sustainability: Science, Practice and Policy, v.3, n.1, p.12-20, 2007. DOI: http://doi.org/10.1080/15487733.2007.11907988

CALZADA, J.; IRANZO, S.; SANZ, A.. Community-Managed Water Services: The Case of Peru. Journal of Environment and Development, v.26, n.4, p.400-428, 2017. DOI: http://doi.org/10.1177/1070496517734020
ESPÍRITO SANTO. Boa Esperança: Programa de assistência técnica e extensão rural PROATER 2011-2013. Vitória, 2011.

ESPÍRITO SANTO. Sistema Integrado de Bases Geoespaciais do Estado do Espírito Santo (GEOBASES). Vitória, 2016.

FIELD, A.. Discovering statistics USING SPSS. 3 ed. SAGE, 2009.

HAIR, J. F.; BLACK, W. C.; BABIN, B. J.; ANDERSON, R. E.; TATHAM, R. L.. Análise multivariada de dados. 6 ed. Porto Alegre: Bookman, 2009.

HELLER, L.; PÁDUA, V.. Abastecimento de água para consumo humano. Belo Horizonte: UFMG, 2006.

HUNTER, P. R.; MACDONALD, A. M.; CARTER, R. C.. Water Supply and Health. PLoS Medicine, v.7, n.11, 2010. DOI: http://doi.org/10.1371/journal.pmed.1000361

HUTCHINGS, P.; CHAN, M. Y.; CUADRADO, L.; EZBAKHE, F.; MESA, B.; TAMEKAWA, C.; FRANCEYS, R.. A systematic review of success factors in the community management of rural water supplies over the past 30 years. Water Policy, v.17, n.5, p.963-983, 2015. DOI:

http://doi.org/10.2166/wp.2015.128

IBGE. Instituto Brasileiro de Geografia e Estatística. Censo Demográfico 2010. Rio de Janeiro: IBGE, 2011.

IBGE. Instituto Brasileiro de Geografia e Estatística.

Estimativas da população residente no Brasil e unidades da federação com data de referência em 10 de julho de 2016 Brasil. Rio de Janeiro: IBGE, 2016a.

IBGE. Instituto Brasileiro de Geografia e Estatística. Pesquisa Nacional por Amostra de Domicílios Contínua (PNAD). Rio de Janeiro: IBGE, 2016b.

KELLY, E.; LEE, K.; SHIELDS, K. F.; CRONK, R.; BEHNKE, N.; KLUG, T.; BARTRAM, J.. The role of social capital and sense of ownership in rural community-managed water systems: 
Qualitative evidence from Ghana, Kenya, and Zambia. Journal of Rural Studies, v.56, p.156-166, 2017. DOI: https://doi.org/10.1016/i.jrurstud.2017.08.021

KLEEMEIER, E.. The impact of participation on sustainability: An analysis of the Malawi rural piped scheme program. World Development, v.28, n.5, p.929-944, 2000. DOI: http://doi.org/10.1016/S0305-750X(99)00155-2

KUMASI, T.; ADANK, M.; SMITS, S.; AGBEMOR, B.; CHIMBAR, T. L.; ATENGDEM, J.. User satisfaction with handpump water services: a synthesis of findings from 3 districts (Akatsi South, East Gonja and Sunyani West Districts). The Hague, 2015.

KUMASI, T. C.; AGBEMOR, B. D.. Tracking user satisfaction of rural water services in northern Ghana. Journal of Water Sanitation and Hygiene for Development, v.8, n.2, p.307318, 2018. DOI: http://doi.org/10.2166/washdev.2018.140

LIKERT, R.. A technique for the measurement of attitudes. Archives of Psychology, v.140, p.5-54, 1932.

MACHADO, A. V. M.; NOGUEIRA, M. T.; ALVES, L. M. C.; QUINDELER, N. S.; SILVA, J. C. D.; ARANHA, P. A. P.; GONÇALVES, R. P.. Assessment of community-managed water systems in rural areas of Espírito Santo, Brazil, using the SIASAR tool. Revista Ambiente \& Água, v.15, n.5, 2020.

MACHADO, A. V. M.; SANTOS, J. A. N.; QUINDELER, N. S.; ALVES, L. M. C.. Critical factors for the success of rural water supply services in Brazil. Water, v.11, n.10, p.1-14, 2019. DOI: http://doi.org/10.3390/w11102180

MAGARA, P.. The paradox of rural water user demand and satisfaction: findings from selected districts in Northern and Mid Western Uganda. IRC Uganda, 2014.

PNUD; IPEA; FJP. Índice de Desenvolvimento Humano Municipal Brasileiro. IPEA, 2013.
RAUTANEN, S. L.; WHITE, P.. Portrait of a successful smalltown water service provider in Nepal's changing landscape. Water Policy, v.20, p.84-99, 2018. DOI: http://doi.org/10.2166/wp.2018.006

SARA, J.; KATZ, T.. Making Rural Water Supply Sustainable, Report on the Impact of Project Rules (UNDP-World Bank Water Sanitation Programme). UNDP - World Bank, 2005.

SIASAR. Manual usuário de SIASAR. SIASAR, 2012.

SIASAR. Protocolo de Campo SIASAR 2.0. SIASAR, 2017.

SPALING, H.; BROUWER, G.; NJOKA, J.. Factors affecting the sustainability of a community water supply project in Kenya. Development in Practice, v.24, n.7, p.797-811, 2014. DOI: http://doi.org/10.1080/09614524.2014.944485

TIGABU, A. D.; NICHOLSON, C. F.; COLLICK, A. S.; STEENHUIS, TAMMO, S.. Determinants of household participation in the management of rural water supply systems: A case from Ethiopia. Water Policy, v.15, n.6, p.985-1000, 2013. DOI: http://doi.org/10.2166/wp.2013.160

UNITED NATIONS. Resolution adopted by the General Assembly on 25 September 2015. Washington, 2015.

UNITED NATIONS. Resolution adopted by the General Assembly on 28 July 2010. Washington, 2010.

WHITTINGTON, D.; DAVIS, J.; PROKOPY, L.; KOMIVES, K.; THORSTEN, R.; LUKACS, H.; BAKALIAN, A.; WAKEMAN, W.. How well is the demand-driven, community management model for rural water supply systems doing? Evidence from Bolivia, Peru and Ghana. Water Policy, v.11, n.6, p.696-718, 2009. DOI: http://doi.org/10.2166/wp.2009.310

WHO. World Health Organization. UN-Water global analysis and assessment of sanitation and drinking-water (GLAAS) 2017 report: financing universal water, sanitation and hygiene under the sustainable development goals. WHO, 2017.

A CBPC - Companhia Brasileira de Produção Científica (CNPJ: 11.221.422/0001-03) detém os direitos materiais desta publicação. Os direitos referem-se à publicação do trabalho em qualquer parte do mundo, incluindo os direitos às renovações, expansões e disseminações da contribuição, bem como outros direitos subsidiários. Todos os trabalhos publicados eletronicamente poderão posteriormente ser publicados em coletâneas impressas sob coordenação da Sustenere Publishing, da Companhia Brasileira de Produção Científica e seus parceiros autorizados. Os (as) autores (as) preservam os direitos autorais, mas não têm permissão para a publicação da contribuição em outro meio, impresso ou digital, em português ou em tradução. 